\title{
Evaluation of a direct immunofluorescence test for diagnosing gonorrhoea
}

\author{
CA ISON,${ }^{*} \mathrm{~K}$ MCLEAN, $\uparrow \mathrm{J}$ GEDNEY,${ }^{*}$ PE MUNDAY,$\dagger \mathrm{D}$ COGHILL,$\dagger \mathrm{R}$ SMITH,$\dagger$ \\ JRW HARRIS, $†$ CSF EASMON* \\ From the Departments of *Medical Microbiology and †Venereology, St Mary's Hospital Medical School, \\ London
}

SUMMARY A new direct immunofluorescence reagent (Syva and Genetic Systems Inc) was evaluated for its ability to detect Neisseria gonorrhoeae in specimens from populations with a high prevalence of the infection. Gonorrhoea was diagnosed by culture in 45 of $105(43 \%)$ urethral specimens from men and 17 of $90(28 \%)$ urethral and 25 of $60(42 \%)$ cervical specimens from women. In men the immunofluorescence test had a sensitivity of $84.4 \%$ and a specificity of $100 \%$; Gram staining gave values of $94 \%$ and $100 \%$, respectively. The sensitivity of the immunofluorescence test could be increased to $89 \%$ by testing duplicate smears. In women the immunofluorescence test had a sensitivity of $65 \%$ and a specificity of $98 \%$ for urethral samples and values of $72 \%$ and $94 \%$, respectively for cervical samples. At both sites the sensitivity of the Gram stain was $40 \%$ and the specificity $100 \%$. The testing of duplicate immunofluorescence smears increased the sensitivity to $76 \%$ for urethral and $88 \%$ for cervical samples.

The diagnosis of gonorrhoea in clinics for sexually transmitted diseases is made on the basis of the Gram stain and culture. Culture of Neisseria gonorrhoeae is regarded as the more sensitive technique, although it may be adversely affected by poor specimen collection, loss of viability during transport from the clinic to the laboratory, and the presence of mutants sensitive to vancomycin. ${ }^{12}$ In clinics for sexually transmitted diseases the Gram stain is used as a rapid test, so that treatment with antibiotics can be given at the patient's first visit. In men the use of Gram stained smears of urethral discharge has a sensitivity of $>90 \%$, but in women the sensitivity is only about $40-50 \% .^{3-6}$

We evaluated a new direct immunofluorescence reagent (Syva Investigational Direct Reagent: developed jointly by Syva and Genetic Systems Inc) among populations with a high prevalence of gonorrhoea. The reagent was compared with Gram staining and culture to assess its efficiency in rapidly diagnosing gonorrhoea in men and women.

\section{Patients and methods}

Specimens were collected from 105 men and 60 women attending the Praed Street Clinic, a large, open access clinic for sexually transmitted diseases. Patients who were particularly likely to have gonor-

Accepted for publication 19 June 1985 rhoea on the basis of history or examiantion were chosen for the initial evaluation of this reagent; thus men were included if they had a urethral discharge or a history suggestive of gonorrhoea and women were included if they had an abnormal vaginal discharge, were sexual contacts of patients with gonorrhoea, or were prostitutes.

\section{COLLECTION OF SPECIMENS}

Specimens from the male urethra and the female urethra were collected with a single, small cotton swab and were used to prepare three slides (two for immunofluorescence and one for a Gram stain) and to inoculate selective and non-selective culture media. Cervical samples were taken using a $10 \mu \mathrm{l}$ disposable loop (Gibco), which was recharged between investigations.

As soon as the specimens needed for this study had been taken all patients were screened for gonor- $N$ rhoea using the standard procedure adopted by the clinic. This differed from that above in that samples from men were taken with a $1 \mu \mathrm{l}$ loop and material was used to make the Gram smear before inoculation on to culture plates.

IMMUNOFLUORESCENCE

For each specimen two coated slides with a single 8 $\mathrm{mm}$ well were used. The swab or loop was used to 
spread the discharge across the well to produce a thin fllm to prevent large amounts of mucus or pus interfering with the reading of the test. The slides were allowed to air dry completely and then were flooded with acetone as a fixative. Delay in the fixation procedure was avoided as it could have caused distorted morphology of the gonococci. When the acetone had evaporated the slides were stored at $-20^{\circ} \mathrm{C}$ until needed.

Before staining, these and the control slides were allowed to equilibrate to room temperature. The immunofluorescence reagent was rehydrated using $2.0 \mathrm{ml}$ distilled water and allowed to warm to room temperature. The reagent $(30 \mu \mathrm{l})$ was placed on each test well and on that of the negative and positive control slides, so that the entire well was covered with reagent. All slides were incubated in a moist chamber for 15 minutes at room temperature. The excess reagent was then removed by agitation in distilled water for 10 seconds. The slides were allowed to dry completely and subsequently mounted. The degree of immunofluorescence and number of fluorescing diplococci on each slide were determined using a fluorescence microscope (Leitz) at $1000 \times$ magnification. Slides were either read immediately or stored at $-20^{\circ} \mathrm{C}$ in the dark and read within 24 hours. A slide was scored positive if one or more pairs of typically shaped diplococci that gave bright apple green fluorescence were present. Both intracellular and extracellular diplococci were considered to be positive. This was in contrast to the Gram stain procedure, in which only intracellular diplococci were regarded as positive. As the Gram stain is non-specific the cellular distribution of cocci provided the only means of distinguishing gonococci.

\section{GRAM STAIN}

Smears were stained by Gram's method using $10 \%$ $(\mathrm{v} / \mathrm{v})$ carbol fuchsine in neutral red as a counterstain. The presence of intracellular Gram negative diplococci was considered to be positive. Extracellular Gram negative diplococci were noted but not considered to be positive.

\section{CULTURE MEDIA}

Neisseria isolation medium containing $36 \mathrm{~g} / \mathrm{l} \mathrm{GC}$ agar base (Difco) and 1\% Isovitalex (BBL) was used in double chamber petri dishes (Sterilin). Non-selective medium was placed in one chamber and the same medium containing vancomycin 3 $\mathrm{mg} / \mathrm{l}$, colistin $1000 \mathrm{IU} / \mathrm{l}$, trimethoprim $5 \mathrm{mg} / \mathrm{l}$, and amphotericin $1.5 \mathrm{mg} / \mathrm{l}$ (selective) in the second chamber. Agar plates were quality controlled using a quantitative technique. Specimens were inoculated directly on to both media in the clinic and immediately stored at $36^{\circ} \mathrm{C}$ in $7 \%$ carbon dioxide for up to four hours. After transportation to the laboratory all specimens were reincubated for up to 48 hours before being discarded as negative.

CONFIRMATORY TESTS

Gram negative cocci from colonies that were oxidase negative were further identified using serum free sugars 7 prepared in $9 \mathrm{~mm}$ petri dishes. $N$ gonorrhoeae was identified by using glucose, but not maltose and sucrose, and by failure to produce $\beta$ galactosidase as measured by the ONPG test.

\section{ANALYSIS OF RESULTS}

Gonorrhoea was defined, for the purposes of this study, by a culture positive for $N$ gonorrhoeae. Sensitivity, specificity, and predictive value of both the immunofluorescence test and the Gram stain, compared with culture, were calculated ${ }^{8}$ as follows:

\section{Sensitivity}

Number of patients with gonorrhoea who were immunofluorescence positive

Total number of patients with gonorrhoea tested

Specificity

Number of patients without gonorrhoea who were immunofluorescence negative

Total number of patients without gonorrhoea tested

Predictive value of a positive test

Number of patients immunofluorescence positive with gonorrhoea

Total number of patients immunofluorescence positive

Predictive value of negative test

Number of patients immunofluorescence negative without gonorrhoea

Total number of patients immunofluorescence negative

READING OF RESULTS

Gram stained and immunofluorescence smears and culture results were done by one observer. The reading was not strictly blind, but each type of test was read separately (the slides in batches), so as to minimise observer bias.

\section{Results}

\section{MEN}

Forty five of the 105 men tested were culture positive for $\boldsymbol{N}$ gonorrhoeae. After a single smear was tested 38 were immunofluorescence positive. None of the 60 culture negative patients was immunofluorescence positive. A second smear from the seven patients who were immunofluorescence 
Sensitivity, specificity, and predictive value of immunoftuorescence test and Gram stain in detecting $\mathbf{N}$ gonorrhoeae in urethral specimens from men and women and cervical specimens

\begin{tabular}{|c|c|c|c|c|c|c|}
\hline & \multicolumn{3}{|c|}{ Immunofuorescence test } & \multicolumn{3}{|l|}{ Gram stain } \\
\hline & \multicolumn{2}{|c|}{ Urethral specimens } & \multirow[t]{2}{*}{ Cervical specimens* } & \multicolumn{2}{|c|}{ Urethral specimens } & \multirow[t]{2}{*}{ Cervical specimens } \\
\hline & $M^{*}$ & $F^{*}$ & & $M \dagger$ & $F$ & \\
\hline \multirow{2}{*}{$\begin{array}{l}\text { Sensitivity } \\
\text { Specificity } \\
\text { Predictive value: } \\
\text { Positive test } \\
\text { Negative test }\end{array}$} & $\begin{array}{l}84 \cdot 4(88 \cdot 9) \\
100(100)\end{array}$ & $\begin{array}{l}65(76) \\
98(98)\end{array}$ & $\begin{array}{l}72(88) \\
94(94)\end{array}$ & $\begin{array}{l}46 \cdot 6(93 \cdot 3) \\
98 \cdot 3(100)\end{array}$ & $\begin{array}{r}36 \\
100\end{array}$ & $\begin{array}{r}40 \\
100\end{array}$ \\
\hline & $\begin{array}{l}100(100) \\
89 \cdot 5(92 \cdot 3)\end{array}$ & $\begin{array}{l}92(93) \\
86(91)\end{array}$ & $\begin{array}{l}90(92) \\
83(92)\end{array}$ & $\begin{array}{l}95 \cdot 4(100) \\
71(95 \cdot 2)\end{array}$ & $\begin{array}{r}100 \\
81\end{array}$ & $\begin{array}{r}100 \\
70\end{array}$ \\
\hline
\end{tabular}

*Figures in parentheses are those obtained after testing duplicate smears (men, $n=7$ ).

$\dagger$ Figures in parentheses are those obtained with routine Gram stain performed in clinic.

negative and culture positive was tested; two of these were immunofluorescence positive.

The Gram smear had been prepared after the immunofluorescence smears. These smears were positive in 21 of the culture positive and one of the culture negative patients. All patients were also tested by routine practice, in which the Gram stain is the first sample taken. These smears were positive in 42 culture positive patients and none of the culture negative patients. The Table shows the sensitivity, specificity, and predictive value for a positive and negative test for both the immunofluorescence and the Gram stain methods.

\section{WOMEN}

\section{Urethral samples}

Seventeen of the 60 urethral samples were culture positive for $N$ gonorrhoeae. The immunofluorescence test was positive in 11 patients after a single smear was tested and in 13 after duplicate smears were tested. The immunofluorescence test was positive in one of 43 culture negative patients.

There was insufficient material on seven of 60 Gram smears to make an assessment. Of the remainder, five of 14 culture positive smears had intracellular Gram negative diplococci.

\section{Cervical samples}

Twenty five of the 60 cervical samples were culture positive for $\boldsymbol{N}$ gonorrhoeae. The immunofluorescence test was positive in 18 after a single smear was tested and in 22 when the duplicate smear was tested. There were also two of 35 culture negative patients who were immunofluorescence positive.

The Gram smear was positive in 10 culture positive patients. The sensitivity and specificity of the Gram smear of urethral and cervical specimens taken by routine procedures were also determined and found to be the same as those determined by the study protocol. The combination of cervical and urethral samples was no more sensitive than cervical samples alone.

\section{Discussion}

Among the male population with a high prevalence of gonorrhoea the new immunofluorescence test was capable of detecting gonococci in most patients There was no advantage, however, over the Gram stain, which already gives an inexpensive, sensitive, and rapid result. The sensitivity of the test in men was improved by the testing of duplicate smears. Both smears from patients with discrepant results had under 10 diplococci per smear, and this suggests that collection of the specimen plays a part in the efficiency of the test and that the more slides that are examined the higher will be the sensitivity of the test. This, of course, would probably also be true for the Gram stain.

The reagent used was a mixture of monocloncal antibodies raised to protein $\mathrm{I}^{9}$ that had been previously tested against strains of $N$ gonorrhoeae isolated in the United States and Sweden. The seven strains from patients whose smears were immunofluorescence negative on initial testing were serogrouped by Dr S Bygdeman (Stockholm). Two of these seven were found to belong to serovars that were not represented in the reagent antibody panel. The sensitivity of the reagent will depend on the inclusion of all suitable antibodies. This means that strains from different geographical areas need to be tested, not only to ensure the initial sensitivity of the reagent but also to monitor new emerging strains. Strains from Britain were not used in the selection of individual monoclonal antibodies for the of immunofluorescence reagent.

Despite the inclusion in the reagent of antibodies $N$ that should have reacted with the remaining five iso- $\omega$ lates, only two of these were positive when retested from pure culture. Unfortunately, the phial of reagent used for retesting was different from that used $\mathscr{D}$ on the smears. A possible explanation is that the activity of some of the monoclonal antibodies was less stable during storage than that of others.

The specificity of the immunofluorescence test 
was $100 \%$ in men. This was the same as that of the Gram stain. It is difficult to imagine a use for this or, indeed, any such reagent in diagnosing urethral gonorrhoea in men given the efficiency of the Gram stain. Both urethral and cervical smears were taken from the women as this is normal practice in this clinic. The Gram stain had a sensitivity of $36 \%$ and $40 \%$ for urethral and cervical smears, respectively. The immunofluorescence test showed a higher sensitivity of $65 \%$ for urethral and $72 \%$ for cervical specimens, which increased to $76 \%$ and $88 \%$, respectively, after duplicate smears were tested.

We think that in this instance false negative results in women were probably due to the way in which specimens were collected rather than the absence of suitable antibodies in the reagent. All the isolates of $\mathbf{N}$ gonorrhoeae from immunofluorescence negative patients available for testing (nine of 13) were immunofluorescence positive when retested from pure cultures. The two duplicate smears from the urethra that were immunofluorescence positive had 10-50 diplococci per smear, and of the four from the cervix, two showed less than five, one showed 10-50, and one showed 50-100 diplococci per smear. Coated slides with two adjacent wells could be used for cervical specimens as multiple samples can be taken when the speculum is in place without any inconvenience to the patient. The performance of this test in women with a high prevalence of gonorrhoea suggests that it could have a useful role in the rapid routine diagnosis of gonorrhoea in women, although it is still less sensitive than culture. The specificity of the immunofluorescence test, unlike the Gram stain, was less than $100 \%$. In one woman the urethral smear was immunofluorescence positive but the culture and Gram stain were negative. The immunofluorescence, culture, and Gram methods, however, were positive with a cervical specimen from this patient, suggesting that the immunofluorescence test gave a true positive result. Two cervical smears from other patients were immunofluorescence positive when all other samples from them were negative. One woman was a sexual contact of a patient with confirmed gonorrhoea and on epidemiological grounds was treated with spectinomycin. The remaining patient was a prostitute who had a history of gonorrhoea but no signs or symptoms of the disease at this visit. She was not treated for gonorrhoea.

A higher sensitivity for the immunofluorescence test among women would be desirable. As the Gram stain is of low sensitivity the immunofluorescence test could be a useful adjunct in a clinic with a transient population, such as this clinic, where rapid diagnosis and treatment are required at the patient's first visit. Despite the test's cost compared with the Gram stain and even culture, time, effort, and money could all be saved by minimising the need for recall visits.
At $98 \%$ the specificity of this test in high risk women is good. Further work is needed to study the specificity in populations with a low prevalence of the disease. Schachter et al showed that even a specificity of $98 \%$ would result in 205 false positive results in 10000 women screened if the prevalence of gonorrhoea was only $1 \% .^{10}$

Detecting gonorrhoea with antibody has also been exploited using an indirect enzyme immunoassay (Gonozyme; Abbott Laboratories). Evaluation of this kit has shown that it is capable of detecting gonococcal antigen in urethral samples from men but has no advantage over the Gram stain. Among women the sensitivity of the test ranged from 85 to $98 \%$ and the specificity was 80 to $97 \% .561011$ The immunofluorescence test may have an advantage for use in clinics for sexually transmitted diseases because of the shorter processing time.

In areas where the resistance of antibiotics to $N$ gonorrhoeae is not a problem tests based on antigen detection may be used to replace culture. When resistant gonococci are regularly encountered, however, these tests can be used only to supplement established cultural techniques.

We thank the nursing staff of the Praed Street Clinic for their help throughout this study.

\section{References}

' Brorson JE, Holmberg I, Nygren B, Seeberg S. Vancomycinsensitive strains of Neisseria gonorrhoeae: a problem for the diagnostic laboratory. Br J Vener Dis 1973;49:452-3.

${ }^{2}$ Mirrett S, Reller LB, Knapp JS. Neisseria gonorrhoeae strains inhibited by vancomycin in selective media and correlation with auxotype. J Clin Microbiol 1981;14:94-9.

${ }^{3}$ Barlow D, Phillips I. Gonorrhoea in women. Lancet 1978;i:761-4.

4 Goodhart ME, Ogden J, Zaidi AA, Kraus SJ. Factors affecting the performance of smear and culture tests for the detection of Neisseria gonorrhoeae. Sex Transm Dis 1982;9:63-9.

${ }^{5}$ Manis RD, Harris B, Geiseler PJ. Evaluation of Gonozyme, an enzyme immunoassay for the rapid diagnosis of gonorrhoea. $J$ Clin Microbiol 1984;20:742-6.

- Burns M, Rossi PH, Cox DW, Edwards T, Kramer M, Kraus SJ. A preliminary evaluation of the Gonozyme test. Sex Transm Dis 1983;10:180-3.

' Flynn J, Waitkins SA. A serum free medium for testing fermentation reactions in Neisseria gonorrhoeae. J Clin Pathol 1972; 25: 525-7.

${ }^{8}$ Vecchio TJ. Predictive value of a single diagnostic test in unselected populations. N Engl J Med 1966;274:1171-3.

- Tam MR, Buchanan TM, Sandstrom EG, et al. Serological classification of Neisseria gonorrhoeae with monoclonal antibodies. Infect Immun 1982;36: 1042-53.

${ }^{10}$ Schachter J, McCormack WM, Smith RF, Parks RM, Bailey R, Ohlin AC. Enzyme immunoassay for diagnosis of gonorrhoea. J Clin Microbiol 1984;19:57-9.

" Danielsson D, Moi H, Forslin L. Diagnosis of urogenital gonorrhoea by detecting gonococcal antigen with a solid phase enzyme immunoassay (Gonozyme ${ }^{\mathrm{TM}}$ ). J Clin Pathol 1983;36:674-7.

Requests for reprints to: Professor CSF Easmon, Department of Medical Microbiology, Wright Fleming Institute, St Mary's Hospital Medical School, London W2 1PG, England. 